\title{
NOTE ON THE ENERGY SCALE OF THE MICHIGAN OSO III ION CHAMBER
}

\author{
RICHARD G. TESKE, PHILIP E. HODGE and SIMON P. WORDEN* \\ Dept. of Astronomy and the McMath-Hulbert Observatory, The University of Michigan, \\ Ann Arbor, Michigan 48104, U.S.A.
}

(Received 11 June, 1971; in final form 10 August, 1971)

\begin{abstract}
The energy scale of the Michigan OSO III soft X-ray ion chamber has been assessed by using realistic theoretical X-ray spectra. Multiplicative factors by which the data may be corrected are proposed. The factors are only slightly temperature-dependent. A test of the proposed energy scale indicates it is still somewhat uncertain.
\end{abstract}

\section{Introduction}

Solar X-radiation has been monitored for many years by means of rocket- and satellite-borne ion chamber detectors. The method of reduction of the ionization current recorded by these devices to an X-ray flux value was early worked out by the pioneers in the field at the Naval Research Laboratory (Kreplin, 1961). In this method, the spectrum shape is fixed by assumption to be that of a black body at some temperature $T$. Upon justifiable observational and theoretical grounds, the temperature defining the spectral shape appropriate to the 8-20 $\AA$ data was selected by them to be $T=2 \times 10^{6} \mathrm{deg}$ (Friedman, 1960). This value has been used ever since, although the data so reduced have been treated carefully in the full realization of their approximate nature (e.g. Kreplin et al., 1962).

The Michigan soft X-ray ion chamber on OSO III was adapted from an NRL design. Solar soft X-ray fluxes were conventionally determined from the telemetered data by adopting the $T=2 \times 10^{6}$ deg grey-body assumption in order to provide a continuity of the observations with those made by NRL. In previous papers concerning our results we have extensively quoted X-ray flux values between $8-12 \AA$ for many observed phenomena on basis of that assumed spectrum. Thomas (1970) concluded that the quoted fluxes were reasonably independent of spectrum, but that they might be systematically a factor of two or three too high.

We have re-investigated the question of the energy scale of the Michigan instrument and find support for Thomas. The results of our calculations provide correction factors which may be applied to the quoted Michigan fluxes. These correction factors depend upon theoretical spectra, which are not yet completely perfected. A test of the proposed energy scale, described below, indicates that the suggested correctionfactors are still to some extent uncertain.

\section{Calculations}

Culhane (1969) has calculated free-free and free-bound continua for thermal plasmas

* Now at the University of Arizona, Tucson, Arizona. 
at a variety of temperatures. We directly adopted his spectra for coronal abundances.

Tucker and Koren (1971) have calculated the power emitted in spectrum lines between 0.5 and $70 \AA$, using coronal abundances, for a wide variety of temperatures. We superposed these emission lines upon Culhane's continua for the calculations which are discussed below.

The ionization current that would be produced when the ion chamber is exposed to the fluxes described by the superposition of emission lines and continua is

$$
\frac{i^{\prime}}{\int N_{e}^{2} \mathrm{~d} V}=\frac{1.078 \times 10^{-8}}{\int N_{e}^{2} \mathrm{~d} V} \int_{0}^{\infty} F(T, \lambda) \varepsilon(\lambda) \mathrm{d} \lambda \mathrm{A} \mathrm{cm}^{3},
$$

where $\varepsilon(\lambda)$ is the band-pass of the detector. The flux of X-rays between 8 and $12 \AA$ would be

$$
\frac{F(8,12)}{\int N_{e}^{2} \mathrm{~d} V}=\frac{\int_{8}^{12} F(T, \lambda) \mathrm{d} \lambda}{\int N_{e}^{2} \mathrm{~d} V} \operatorname{erg~cm~s}{ }^{-1} .
$$

In carrying out our actual data reductions, the quoted fluxes were calculated from (c.f. Kreplin, 1961)

$$
E(8,12)=7.75 \times 10^{8} i \mathrm{erg} \mathrm{cm}^{-2} \mathrm{~s}^{-1}
$$

In Table I we list the electrical currents which would be produced (per $N_{e}^{2} V$ ) by exposing the ion chamber to a 'realistic' flux of X-rays (from Equation (1)), the 'realistic' flux $F(8,12)$ as calculated from Equation (2) and the flux value that would have been quoted by us had we observed the electrical current $i$ ' (from Equation (3)). In the last column is given the correction factor which converts the quoted flux to the 'realistic' flux.

These correction factors indicate that the quoted energy scale for the Michigan data is too high by about $30 \%$ for the quiet Sun and by about $40 \%$ for phenomena that reach $T_{e}=20 \times 10^{6} \mathrm{deg}$. In general, however, the relative fluxes quoted from our data

\section{TABLE I}

Proposed correction factors for Michigan OSO III ion chamber data

\begin{tabular}{lllll}
\hline $\log T_{e}$ & \multicolumn{1}{c}{$i^{\prime}$} & $\frac{F(8,12)}{\int N_{e}^{2} \mathrm{~d} V}\left(\mathrm{erg} \mathrm{cm} \mathrm{s}^{-1}\right)$ & $\frac{E(8,12)}{\int N_{e}^{2} \mathrm{~d} V}\left(\mathrm{erg} \mathrm{cm} \mathrm{s}^{-1}\right)$ & $\begin{array}{l}\text { Correction } \\
\text { factor }\end{array}$ \\
\hline 6.3 & $2.99 \times 10^{-62}$ & $1.82 \times 10^{-53}$ & $2.32 \times 10^{-53}$ & 0.78 \\
6.5 & $3.23 \times 10^{-61}$ & $1.92 \times 10^{-52}$ & $2.50 \times 10^{-52}$ & 0.77 \\
6.7 & $1.26 \times 10^{-60}$ & $7.57 \times 10^{-52}$ & $9.72 \times 10^{-52}$ & 0.78 \\
7.0 & $3.59 \times 10^{-60}$ & $2.08 \times 10^{-51}$ & $2.78 \times 10^{-51}$ & 0.75 \\
7.3 & $2.42 \times 10^{-60}$ & $1.30 \times 10^{-51}$ & $1.87 \times 10^{-51}$ & 0.70 \\
\hline
\end{tabular}


are independent of spectrum shape, between $2 \times 10^{6} \leqslant T_{e} \leqslant 20 \times 10^{6} \mathrm{deg}$, to within $12 \%$.

Horan (1970) and other NRL observers (Kahler et al., 1970) have found that at the $\mathrm{X}$-ray maximum in flares the appropriate $T_{e}$ for soft X-rays is in the neighborhood of $10 \times 10^{6}$ to $20 \times 10^{6} \mathrm{deg}$. Thus the relative flux amplitudes quoted by us for flares are perhaps correct to about $7 \%$ or better, and time-integrals of energy relatively correct to about $10 \%$.

We especially note that the instrumental ion chamber current produced per unit emission measure on the Sun is nearly independent of temperature (to within $50 \%$ ) between $10 \times 10^{6} \leqslant T_{e} \leqslant 20 \times 10^{6} \mathrm{~K}$. Thus, during flares the instrument responded chiefly to variations of emission measure. Relative flux data quoted for flares (e.g. Teske, 1969; Thomas and Teske, 1971) thus principally depict the differences in emission measure which characterize the soft X-ray source in various flares.

These conclusions support Thomas's (1970), which were based upon semiartificial spectra. The corrections proposed here, however, require a test of their acceptability.

\section{A Test of the Calculated Response}

The above calculations may be tested upon published observations that were made at the same time as our own. One such test may be made using the spectrum recorded on 9 Nov., 1967, by a proportional counter and reported by Culhane et al. (1969). This spectrum is shown as the full line in our Figure 1. At the time of their observation, our OSO III ion chamber current was $2.58 \times 10^{-12} \mathrm{~A}$.

Culhane et al. used Culhane's (1969) continuum calculations and fitted their observed spectrum by a coronal model containing two components described by (i) a component at $5 \times 10^{6} \mathrm{deg}, N_{e}^{2} V=1.7 \times 10^{47} \mathrm{~cm}^{-3}$ and (ii) a component at $3 \times 10^{6} \mathrm{deg}$, $N_{e}^{2} V=5 \times 10^{48} \mathrm{~cm}^{-3}$. Their model failed to fit the observed spectrum longward of $6 \AA$ wavelength, and they concluded that there must have existed 'substantial volumes of plasma at temperatures below $3 \times 10^{6}$ deg'.

We have attempted to fit Culhane et al.'s observed spectrum by a three-component model based upon the theoretical continua and lines utilized in this study. A constraint upon the model is that it must reproduce the observed ion chamber current. The parameters of a three-component model which fits the data are listed in Table II and the resulting fit to the observed spectrum, averaged over one-angström intervals, is shown in Figure 1. Although the spectrum fit is good and the model reproduces the observed ion chamber current, an emission measure of $7.1 \times 10^{49} \mathrm{~cm}^{-3}$ is required.

\section{TABLE II}

Three-component model to fit spectrum by Culhane et al. (1969)

\begin{tabular}{lcl}
\hline $\log T_{e}(\operatorname{deg} \mathrm{K})$ & $N_{e}^{2} V\left(\mathrm{~cm}^{-3}\right)$ & Ion chamber current (A) \\
\hline 6.3 & $7 \times 10^{49}$ & $2.09 \times 10^{-12}$ \\
6.5 & $1 \times 10^{48}$ & $0.32 \times 10^{-12}$ \\
6.7 & $1.5 \times 10^{47}$ & $0.19 \times 10^{-12}$
\end{tabular}




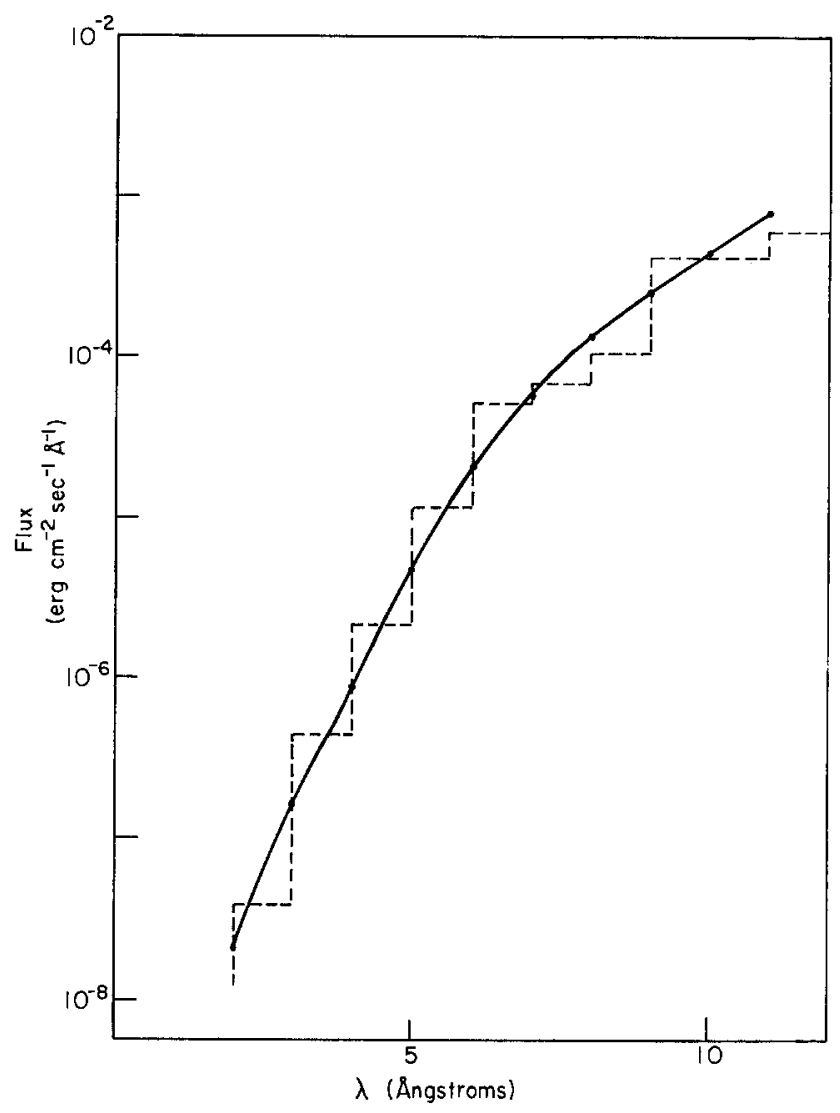

Fig. 1. The full line is the X-ray spectrum observed 9 Nov., 1967, by Culhane et al. The dashed histogram is the theoretical spectrum fitted to it using the energy scale for the Michigan ion chamber which is proposed in this paper.

This seems rather excessive. From the model of an active region by Christiansen et al. (1960), we obtain a representative columnar electron surface density squared in active regions of

$$
\int N_{e}^{2} \mathrm{~d} h \sim 9 \times 10^{28} \mathrm{~cm}^{-5} .
$$

On 9 Nov., 1967, the total plage area was $15300 \times 10^{-6}$ of a solar hemisphere, yielding

$$
\sum A \int N_{e}^{2} \mathrm{~d} h \sim N_{e}^{2} V \sim 4 \times 10^{49} \mathrm{~cm}^{-3} .
$$

We consider this test of the proposed energy scale for our instrument to be a partial success, since the energy required to explain the ion chamber current leads directly through the proposed calculations to a good fit of the observed spectrum. However, the required emission measure appears to be about $80 \%$ larger than an acceptable 
value. If we wish to make the derived emission measure match that predicted from Christiansen's model, we must increase the 'realistic' fluxes per unit emission measure of Table I above those given by the theoretical spectra which were employed, thereby increasing the correction factors and making them closer to unity.

\section{Conclusions}

Use of 'realistic' $\mathrm{X}$-ray spectra rather than the standard grey-body approximation in calculating ion chamber response leads to the conclusion that flux values quoted for the Michigan OSO III ion chamber are semi-independent of the shape of the thermal spectrum over a temperature range $2 \times 10^{6} \leqslant T_{e} \leqslant 20 \times 10^{6} \mathrm{deg}$, to within $12 \%$, on a relative energy scale. For flares in the range $10 \times 10^{6}$ to $20 \times 10^{6} \mathrm{deg}$, the relative flux values are correct to $7 \%$.

A multiplicative factor which corrects the quoted fluxes to the 'true' fluxes is temperature-dependent, and may be calculated from a suitable theoretical spectrum. The correction factors cannot yet be stated with certainty, but they lie in the range 0.7-1.0. If Culhane's (1969) continua and Tucker and Koren's (1971) emission line powers correctly represent the Sun, the correction factors of Table I may be applied directly.

\section{Acknowledgements}

We are indebted to W. H. Tucker and M. Koren for sending us a copy of their calculations prior to publication.

\section{References}

Christiansen, W. N., Mathewson D. S., Pawsey, J. L., Smerd, S. F., Boischot, A., Denisse, J. F., Simon, P., Kakinuma, T., Dodson, H. W., and Firor, J.: 1960, Ann. Astrophys. 23, 75.

Culhane, J. L.: 1969, Monthly Notices Roy. Astron. Soc. 144, 375.

Culhane, J. L., Sanford, P. W., Shaw, M. L., Phillips, K. J. H., Willmore, A. P., Bowen, P. J., Pounds, K. A., and Smith, D. G.: 1969, Monthly Notices Roy. Astron. Soc. 145, 435.

Friedman, H.: 1960, 'The Sun's Ionizing Radiations', in Physics of the Upper Atmosphere (ed. by J. A. Ratcliffe), Academic Press.

Horan, D. M.: 1970, Dissertation, Catholic University of America.

Kahler, S. W., Meekins, J. F., Kreplin, R. W., and Bowyer, C. S.: 1970, Astrophys. J. 162, 293.

Kreplin, R. W.: 1961, Ann. Geophys. 17, 151.

Kreplin, R. W., Chubb, T. A., and Friedman, H.: 1962, J. Geophys. Res. 67, 2231.

Teske, R. G.: 1969, Solar Phys. 6, 193.

Thomas, R. J.: 1970, Dissertation, University of Michigan.

Thomas, R. J. and Teske, R. G.: 1971, Solar Phys. 16, 431.

Tucker, W. H. and Koren, M.: 1971, Astrophys. J. 168, 283. 